\section{$\underset{\substack{\text { hommes } \\ \text { \& migrations }}}{ }$}

\section{Hommes \& migrations}

Revue française de référence sur les dynamiques

migratoires

$1311 \mid 2015$

Femmes et migrations

\title{
Figures (littéraires) de la « femme immigrée »
}

\section{Mustapha Harzoune}

\section{(2) OpenEdition \\ Journals}

\section{Édition électronique}

URL : http://journals.openedition.org/hommesmigrations/3398

DOI : 10.4000/hommesmigrations.3398

ISSN : 2262-3353

\section{Éditeur}

Musée national de l'histoire de l'immigration

\section{Édition imprimée}

Date de publication : 1 juillet 2015

Pagination : 184-185

ISBN : 978-2-919040-32-2

ISSN : $1142-852 X$

\section{Référence électronique}

Mustapha Harzoune, «Figures (littéraires) de la « femme immigrée » », Hommes \& migrations [En ligne], 1311 | 2015, mis en ligne le 23 février 2016, consulté le 15 septembre 2020. URL : http:// journals.openedition.org/hommesmigrations/3398 


\section{LITTÉRATURE}

\section{FIGURES (LITTÉRAIRES) DE LA "FEMME IMMIGRÉE"}

par MUSTAPHA HARZOUNE

A vec leur entrée dans l'espace de la migration, les femmes migrantes font irruption dans la fiction, ouvrant de nouveaux horizons narratifs. Pour en juger, voici un caléidoscope de la femme immigrée dans la production littéraire contemporaine.

La littérature, à l'instar des études sociologiques ou historiques, n’a pas été hospitalière à la figure de l'immigrée. Ou tardivement. Prérogative du genre ou antériorité de la présence, l'immigré, l'ouvrier et le père, ses silences et meurtrissures inspiraient - tourmentaient parfois - davantage la plume et l'âme des écrivains. Pour autant, évoquer l'immigration au féminin demande de préciser de qui l'on parle. Ici aussi, les mots sont des pièges. "Femme immigrée"! "immigré”, "issus de l'immigration", "clandestin sans-papiers", "beur"... autant de vocables qui prétendent tout dire et ne révèlent rien. On ne cache pas le soleil avec un tamis. Que dissimulent alors ces deux mots, "femme immigrée" ? Une réfugiée espagnole fuyant le franquisme' ? Une Argentine en quête d'asile dans les années $1970^{2}$ ? Des Chinoises des temps présents ? Celles des ateliers clandestins ou des salons esthétiques ${ }^{3}$ ? Ou celles qui arpentent le boulevard de Belleville à Paris ? S'agit-il d'une Algérienne mère de famille préoccupée du qu'en-dira-t-on ${ }^{4}$ ? À moins qu'elle ne soit africaine et sans papiers ${ }^{5}$, géorgienne au soir de sa vie ${ }^{6}$ ou vénérable arménienne gourmande de tiropitakias et autres beureks?. Elle peut être épouse et mère, cantonnée aux tâches domestiques ou salariée qui élargit le cercle de l'émancipation. Parle-t-on du commerce des cœurs ? De sexualité ? Celle tarifée et esclavagisée ${ }^{8}$ ou du désir qui naît avec l'Autre ? Et pour être moderne, l'immigrée, figure maternelle, laborieuse, sexuelle ou sentimentale, soumise ou rebelle, fut aussi d'un autre temps. Celui, par exemple, d'Albert Cohen ou de Romain Gary. II en est même qui, sans avoir jamais quitté leur patelin, ont souffert de l'exil. Tandis qu'au premier âge de l'immigration, l'aède expatrié s'en allait "louer les filles kabyles' ${ }^{10 "}$ restées au pays, les mêmes chantaient : "Qui t'a dit, mon frère, de suivre / Les émigrants dans leur exil? / Ton absence me parait si longue! /Tu m'as laissée toute seule dans mon lit"."

Il faut donc circonscrire. Préciser de qui l'on parle. Les auteur(e)s d'abord : ils sont d'aujourd'hui et le plus vieux des romans ici évoqués (et sur ce thème) n'a pas dix ans. Point de prétention à l'exhaustivité mais un tour d'horizon, au petit bonheur des lectures. Les femmes ensuite : elles composent un tableau impressionniste d'une immigration au féminin, femmes vieillissantes ou nouvelles figures, par les métiers ou les aspirations, par

\footnotetext{
1. Léonord de Récondo, Rêves oubliés, Paris, Sabine Wespieser, 2012. 2. Laura Alcoba, Le Bleu des abeilles, Paris, Gallimard 2013. 3. Sylvain Pattieu, Beauté Parade, Paris, Plein Jour, 2015. 4. Faïza Guène, Un homme, ça ne pleure pas, Paris, Fayard 2014.

5. Fadéla Hebbadj L'Arbre d'ébène, Paris, Buchet-Chastel, 2008.6. Kéthévane Davrichewy, La mer Noire, Paris, Sabine Wespieser, 2010. 7. Martin Melkonian, Arménienne, Paris, Maurice Nadeau, 2012 8. Max Lobe, 39 rue de Berne, Carouge, Zoé, 2013. 9. Saïd Mohamed, Ciel de lune, Paris, L’Arganier, 2007. 10. "Je m'en vais louer les filles kabyles", chanté par Cheikh El Hasnaoui 11. Chant traditionnel de Kabylie, Samia Messaoudi et Mustapha Harzoune, Paroles kabyles, Paris, Albin Michel, 2000.
} 
les pays d'origine - elles sont maghrébines, subsahariennes, sudaméricaines, asiatiques... La mondialisation de l'immigration en boubou, en pancho, en qipao, en gandoura et autre ao dai.

\section{Figures de chibanias}

Au commencement était la mère. L'imagerie en retient une figure mêlant l'effacement, la subordination, si ce n'est la soumission. Le pendant de la domination masculine. L'invisible féminin de l'invisible masculin. "Le temps d'une traversée, elles étaient devenues des étrangères ${ }^{12}$ ", ces femmes venues rejoindre leur immigré de mari. "Les pleurs sans bruit, la main sur la bouche ${ }^{13}$ ", et les voilà arrivées. Au pied du mur. Dans une chambre minable d'un hôtel minable. Le Bleu des abeilles de Laura Alcoba ${ }^{14}$ évoque cette universelle déconvenue. Ici, à défaut du barrio latino, la réfugiée argentine s'installe au Blanc-Mesnil, ses vêtements viennent du Secours catholique et l'appartement, modeste, est partagé avec une autre réfugiée. "Elles étaient ensemble à l'université, toutes les deux faisaient des études d'histoire. Alors, quand elles se sont retrouvées par hasard, à Paris, après les disparitions, la peur et les morts, elles se sont naturellement serré les coudes." La réfugiée passe des bancs de l'université aux boulots de fortune. Déclassement social obligé. Déception et désillusion donc. Et parfois, la honte du père : "Quand il a posé les yeux sur moi,j'ai vu quelque chose qui ressemblait à de la honte. II semblait s'excuser auprès de moi qui n'avais que six ans. Aujourd'hui, quand je repense à ce regard, je dois me retenir pour ne pasfondre en larmes's."

Débarquée, avec ou sans progéniture, I'horizon peut se réduire à l'espace domestique. "Ma mère, elle, ne fait rien. En général après notre départ elle lave la vaisselle, donne un dernier coup de serpillère, s'assoit à la table de la cuisine, à la même place que le matin, puis lentement touille son café noir'." Elle "sèche d'ennuir".

$\mathrm{Si}$, pour certaines, le cercle de l'autonomie va s'élargissant, pour d'autres, la figure maternelle trône dans son salon. Ou dans sa cuisine. Dans ce temple domestique par elle sacralisé. Elle y officie telle une vigie. Gardienne sourcilleuse des traditions, elle débusque le sacrilège et prévient l'innovation impie. Au sein de chaque famille passe une ligne, floue, instable, qui sépare une certaine tradition d'une certaine modernité ; l'invariant du nouveau ; la reproduction des rapports de domination de leur contestation. Dans Un homme ça ne pleure pas de Faïza Guène ${ }^{18}$, chez les Chennoun, la mère campe cette gardienne du temple. Elle s'offusque, gronde, tempête, sanglote, se lamente et brandit l'arme de la "culpabilisation" pour faire entrer dans le rang mari et progéniture. "Tout se négocie chez nous, tout se traite, le bakchich sentimental'19", écrit aussi Samira Sedira. Difficile de résister. Sauf à rompre, comme Dounia, l'aînée de la fratrie. Ou à se trimballer, quand on est l'unique garçon de la reine mère, une encombrante laxophobie, née de la culpabilité reçue en héritage et d'un trop-plein d'amour maternel et castrateur. C'est le bal des injonctions normatives et contradictoires, le jeu des fidélités et des trahisons, le bastringue des identités qui inventent et réinventent un rapport au monde et à soi.

Mais, quand c'est le mari qui, au nom de l'honneur et de sa moustache, campe le cerbère, l'ordre peut devenir autrement brutal pour l'épouse et mère "C'est de ta faute, crie le fils au père au-dessus du corps sans vie de la mère, tu lui avais interdit de sortir! Comment t'as pu penser qu'elle supporterait cette vie ?" Ici, la "maman a été de ces folles inoffensives, sans haine, qui n'ont rien tué d'autre qu'ellesmêmes ${ }^{20}$." Dans Le Royaume sans racines2i, Sema Kiliçkaya dénonce ce sort fait aux femmes. Ici, les émigrées sont de Turquie mais les mentalités barbotent dans le même cousinage. Elle raconte le 


\section{LITTÉRATURE}

désir de femmes souvent soustraites, leurs désillusions et les ruptures par la transgression, la séparation - ou le suicide! Le Royaume sans racines montre comment la liberté de ces femmes immigrées participe à - et conditionne - la ruine des enfermements communautaires et religieux.

\section{Bifurcations générationnelles}

Une autre peur hante la figure, littéraire et maternelle, de l'immigrée : celle de l'éloignement des générations. Elle s'affiche, depuis le refus hystérisé jusqu'à l'acceptation silencieuse. Ce risque se perd dans les contours d'un rêve, il disparaît derrière les premières brumes de l'illusion. "C'était cela l'Europe, une double journée de travail, une vie sociale réduite à néant, les quolibets de ceux qui avaient l'avantage d'être du coin, un ciel bas et pesant et des enfants que l'on perdait, soit parce qu'ils étaient malencontreusement tués, soit parce qu'ils dérivaient doucement hors de votre culture. Est-ce que les candidats au départ savaient tout cela? Quand bien même l'eussent-ils su, cela les aurait-il empêchés de continuer à rêver? Certainement pas. Car c'était ça, l'Europe : un rêve $e^{22 . " ~}$

Et quand ce "rêve" s'en vient heurter le "dur pépin de la réalité", l'exil devient, jusque dans la chair, une "régression"23". C'est l'heure où les épouses et mères découvrent les inévitables bifurcations générationnelles qui fertilisent toutes migrations. Pourtant, l'envol des plus jeunes vers "/a découverte du monde" bute sur la "crainte" atavique de "tous les parents immigrés": "voir son enfant gagné à l'autre culture et perdu à la sienne". De cette "peur viscérale naitrait la tentation de la geôle protectrice". "Pour protéger sa descendance, on allait ériger des murs, bâtir des forteresses. La famille allait devenir cellule24." Dans Zone cinglée,
Kaoutar Harchi décrit la lente et tragique décomposition d'une Cité. Sur fond de déraison, de folie, d'interdits et de frustrations sexuels, les femmes et mères s'y organisent pour que cessent les violences qui voient leurs fils tombés un à un. Elles se mobilisent pour une "cause" incertaine : "protéger la Cité", quitte à refermer sur elles les grilles invisibles de ces "zones cinglées" les grilles d'une autre "cellule".

Mais la mère est aussi celle qui entrouvre la porte, quitte à sangloter en secret. Le "fiston prometteur" de Victoria ${ }^{26}$ l'Arménienne pourrait être le rejeton de Taos, la sage Kabyle. Dans La Trempe, Magyd Cherfi parle de sa mère qui "étouffait dans son impossibilité à nous porter secours". "Elle sétait effacée au fil du temps pour nous faire plus de place avec en filigrane la prétention de nous sortir de la mouise." Et cet aveu d'impuissance : "L'amour des pauvres n'a pas de mesure. (...) Maman nous a aimés pour qu'on lui ouvre des portes trop grandes pour nous, pour qu'on allume la lumière alors que l'interrupteur était trop haut27." Samira Sedira évoque avec la même tendresse cette figure maternelle, attentive à l'instruction, fière de l'ascension sociale de sa fille, "mais dans le même temps elle craignait que je lui échappe. L'instruction, une amie-ennemie, son ravisseur d'enfant". La relation, nourrie d'une mutuelle attention, peut voir s'inverser l'ordre de la transmission: "Le jour où je lui ai appris que j'avais rencontré quelqu'un que j'aimais et qui maimait, j'avais vingt-deux ans. Un sourire a tremblé sur ses lèvres. Elle m'a demandé en tordant son petit mouchoir brodé entre ses doigts, C'est vrai ce qu'on dit... ? qu'on a le coeur qui bat plus fort... ?"

Et puis vient la mort. Dans La Guerre des fleurs de Nadia Berguet ${ }^{28}$, huit enfants se retrouvent au chevet de leur mère mourante. La construction laisse entendre les monologues intérieurs qui rythment les cinq temps du roman. C'est l'heure 
nias', nouveaux visages de la vieillesse immigrée $^{32}$ ". La noria des générations efface ces personnages de fiction ou ces ombres discrètes, témoins d'un autre âge de l'immigration ; d'autres femmes s'en viennent peupler les pages des littérateurs. Pour tout chambouler, brouiller les repères, s'arroger plus d'importance. Et parfois le premier rôle. Partir n'est plus seulement une décision masculine. Migrer devient une initiative de femmes, provoquée par les opportunités professionnelles qui s'offrent ailleurs, et uniquement à elles. Partir aussi pour s'émanciper, conquérir, sur les siens et sur le sort, une liberté nouvelle. Partir non plus comme une promesse mais comme un soulagement.

Gare de Lyon, Paris, 1992. (c) Charles Carrié.

des bilans. Les vies, les blessures et les non-dits s'exposent. Histoire de famille. Histoire d'une famille française aux origines ensoleillées par la figure maternelle. Mais, à l'heure des obsèques, les enfants se voient dépossédés du corps de leur mère. La défunte est reprise par le groupe et les religieux. Jamal Mahjoub29 et Azouz Begag30 avaient déjà rendu compte de cette dépossession.

\section{Partir pour l'aube, entrer dans la nuit}

Les chibanias (celles qui ont les "cheveux blancs") vieillissent donc. Dans le cocon plus ou moins douillet de la famille. Le regard tourné vers le lointain ou vers les proches. Ou veuves, et comme abandonnées. Oubliées. II faudrait un Abdelkader Djemaï pour dresser le portrait de ces "'chiba-
Dans L'Arithmétique des dieux, Katrina Kalda33 raconte le destin des Raud, une famille estonienne ayant traversé la guerre, l'occupation allemande, les déportations staliniennes et, enfin, l'avenir radieux de l'eschatologie soviétique. Kersti, la mère de Kadri, la narratrice, ne supporte plus sa famille. Pour ne pas étouffer, elle décide de s'esbigner en 1989. Elle embarque avec sa fille pour Paris, laissant derrière elle l'Estonie encore sous domination soviétique, le quotidien terne, sa méchante belle-mère, son époux, ramolli par la vodka et l'excès de nicotine et obsédé par le rangement. Paris, "la ville de tous les possibles", allait accueillir les bras grands ouverts la jeune femme, francophile, professeur de piano, à la carrière "fichue". II n'en sera rien. Comme tant d'autres, Kersti découvre que la Ville lumière est aussi ville de misère. Pour "viure sa propre vie", il va falloir en payer le prix.

Toutes ne partent pas pour "vivre [leur] propre vie". Elles sont nombreuses à s'aventurer dans la nuit noire de l'exil, doublement "vulnérables ${ }^{34}$ " parce 


\section{LITTÉRATURE}

qu'elles doivent subir les dangers et les hommes, sans pouvoir se plaindre. Ce sont alors des ambassadrices, un capital humain exporté pour assurer l'avenir des leurs. L'avenir d'un enfant, resté au pays ou emmené avec soi. Dans L'Arbre d'ébène de Fadéla Hebbadj35, Mama a promis à son fils Nasser qu'un jour ils auront un réfrigérateur, un grand lit et un homme, "l'arbre d'ébène" qui les protégera. Pour le moment, réfugiés dans un squat à Paris, ils n'ont qu'un sac de couchage pour réchauffer leurs solitudes. Alors ? Promesses ? Mensonges ? "Tu m'as menti, depuis ce voyage. Là-bas on était mieux, maintenant si tu meurs qui va s'occuper de moi ?" s'inquiète Nasser. Là-bas, cétait en Afrique. Après l'enfer d'un périple qui, du Mali à Marseille, a été rythmé par des drames, des trahisons, des viols, d'autres dangers guettent une mère africaine malade et son rejeton : la peur de la police, les squats et les foyers douteux, l'hostilité et le rejet... C'est aussi par ses marges qu'une société se révèle à elle-même ${ }^{36}$. "Dans notre pays, on les accueille avec le respect et l'hospitalité, ils viennent avec le sourire et repartent avec de bons souvenirs, ici ils nous accueillent avec des matraques et nous font vivre des cauchemars comme des criminels...", dit Mama. La mère et son fiston dévoilent les peurs, la méchanceté, le culte de l'argent, "les portemonnaie sans valeur "... Fadéla Hebbadj écrit un livre sur une mère et son fils, sur le dévouement et même le sacrifice de l'une et l'amour de l'autre. "Nasser est mon fils, et il a ses lectures. II m'a suivie en enfer, je tiens à lui trouver une place ailleurs que chez nous. II faut qu'il rencontre le monde loin des nôtres..." "Moi, j'ai la chance d'avoir une mère comme celle de mon livre", celui de Romain Gary, celui de La Promesse de l'aube, dit Nasser.

Ce que décrit Max Lobe ${ }^{37}$ est une autre figure de l'émigration au féminin, celle de la domination et de l'exploitation. Ici, la décision de partir est prise par d'autres et scellera le destin de l'émigrée-immigrée pour la vie. Dans 39 rue de Berne, la jeune Mbila est envoyée en Europe par son "Tonton". Elle ignore qu'elle est confiée à un réseau de passeurs, un réseau de "feyman" (d'escrocs), les Philanthropes-Bienfaiteurs, histoire qu'elle "devienne quelqu'un" et expédie du "mbongo" (de l'argent) à la famille. Émigrer, c'est aussi porter et supporter - la charge d'une "grande mission". À 16 ans, Mbila se trouve piégée, plombée par une dette qu'elle n'a pas contractée. Son parcours est dès lors fléché : deux ans de prostitution pour rembourser, suivi d'un mariage blanc avec un "mariageur" pour obtenir des papiers, le tout agrémenté d'une participation obligée à un trafic de cocaïne !

\section{Au boulot !}

Mbila aura un fils, Dipita. Très jeune, il devient I'"associé" et le "chargé de communication" de sa prostituée de mère mais toujours, à ses yeux, "princesse bantoue". II raconte les passes et les visites interlopes, la rue de Berne à Genève où les tapineuses tapinent. Les membres de l'AFP, I'“Association des Filles des Pâquis", club des prostitués du quartier des Pâquis, se retrouvent chez Mbila autour d'un verre ou d'un gros joint pour partager "leurs trucs de wolowoss-là" (prostitués). Il y a Bélen, la Bolivienne, Maïmouna, la Rwandaise, Tran-Hui, la Thaillandaise, Charlotte, la Nigérienne et Mbila, la Camerounaise... "On rit (...) parce que dans le rire se cache peut-être un peu d'espoir ${ }^{38}$." C'est le comité central du tapinage globalisé et de l'esclavage moderne.

D’autres grossissent l'armée de réserve du capitalisme mondialisé. À elles les nouveaux boulots : nounou, femme de ménage, aide aux soins et à la personne, commerces en tout genre. Dans Beauté 
Parade, Sylvain Pattieu 39 pousse la porte d'un salon de beauté de la capitale. II s'agit d'un de ces salons du quartier Château-d'Eau, onglerie et cheveux, où s'affairent des femmes, immigrées, sans papiers souvent, invisible piétaille de cette "armée de réserve" dans laquelle puise une économie vorace. Ce sont certes des "engagées volontaires, mais choisit-on vraiment, quand on vient du mauvais côté du monde40 ?". Elles sont six femmes, Lin Mei, Fengzhen, Yanping, Souqin, Madissou et Adja, et un homme, Gang. Jusqu'à ce jour où elles se sont mises en grève, l'Afrique et l'Asie s'ignoraient. Les langues, les cuisines, les mémoires comme les espoirs glissaient, indifférents les uns aux autres. Elles sont parties en lutte comme d'autres embarquent sur un rafiot de fortune. Ces travailleuses de l'ombre ont décidé de dire non, d'occuper les lieux. De se faire entendre. De revendiquer le droit à un peu de dignité et de liberté. Être payées. Être régularisées. Les femmes irriguent désormais de leur énergie, de leur sueur, de leurs espoirs et déceptions, de leur sang aussi, les artères enchevêtrées de I'humanité migrante. Ce sont ici des "femmes métamorphes" qui "obtiennent ou acquièrent la liberté de mouvement, du corps et de l'esprit 4l'. Pendant qu'on anesthésie le chaland d'islamosornettes, lustrant du même coup le monstrueux "quart d'heure de célébrité" de ceux qui, parce que l'on a jamais parlé d'eux, s'en vont souiller de leurs excréments la fontaine du village, on en oublie celles qui par leur travail, leur quête de liberté, leurs luttes contribuent à renforcer l'espace de tous et de chacun, à harmoniser (et ensoleiller) le tableau collectif.

Dans Des Bédouins dans le polder. Histoires tragicomiques de l'émigration ${ }^{42}$, Fouad Laroui conte les heurs et malheurs des enfants du Rif transplantés en terre batave. Si côté féminin, Fouad Laroui rapporte avec quelque malice la recherche surréaliste d'emploi d'un "jéroboam ambulant", entendre une femme voilée de pied en cap, il montre aussi l'effet de l'ouverture d'une auto-école pour femmes musulmanes au grand dam de la gent masculine et machiste, pedzouille du volant et oublieux de ce temps où “'épouse du Prophète, Khadija, dirigeait elle-même sa caravane à travers le désert d'Arabie. Et qui peut mener cent chameaux peut bien conduire une quatre-chevaux, non ?" dixit Fouad Laroui rappelant Assia Djebar43, qui déjà s'en était allée revisiter la Médine du prophète pour rabattre le caquet de quelques contemporains phallocrates.

Ces femmes immigrées peuvent donc en remontrer aux gardiens de l'ordre et de l'honneur à moustache. Elles peuvent aussi épingler de petits marquis jaloux, eux, d'une préséance tout occidentale. Dans Une enfant de Poto-Poto, Henri Lopes 44 évoque la migration d'étudiantes congolaises. "Le fleuve détourné " de la "Ndependa" - l'indépendance version locale -, le pays est devenu "insupportable" à ces jeunes filles qui ne rêvent que de partir étudier à l'étranger et notamment à Paris, "partir à la Sorbonne, comme Villon, Césaire et Senghor". Les arcanes de l'administration congolaise mèneront la jeune Kimia aux USA. Après les premières larmes et les déconvenues de l'exil, Kimia, l'immigrée congolaise, entreprend sa "métamorphose" culturelle. Elle s'affranchit de l'illusion des racines et des origines - "aucune fonction algébrique, aucun programme d'ordinateur ne rend compte des destins" - pour célébrer le primat des "vivants" sur les fausses "authenticités" et le pseudo "patrimoine identitaire". Universitaire et écrivaine de renom, elle s'emploie à "congoliser le roman". C'est une autre facette de l'immigration au féminin qui se donne à voir : "ll aura suffi d'une génération pour que nous nous hissions à la hauteur des jeunes Européens ou Américains. Non, aucun devin, aucun programme d'ordinateur n'aurait su prévoir que les négrillons enfantés par des 


\section{LITTÉRATURE}

analphabètes sur la natte, dans des ajoupas, manieraient un jour sans anicroche les langues de Shakespeare, de Goethe et de Pouchkine, jongleraient avec les algorithmes, traduiraient Eschyle et Suétone, piloteraient des avions à réaction, construiraient des routes, des immeubles, des ponts, dirigeraient des équipes dans des salles d'opérations ${ }^{46}$."

\section{Entre malentendus et apprivoisement}

Avec l'arrivée de la femme dans l'espace de la migration, les corps réintègrent la chronique littéraire. Des corps qui parlent. Disent. Des corps désirables. Désirants. Convoités. Aimés. Fantasmés. Souillés. Meurtris. Violentés. Corps objet. Corps sujet. Corps capital. Outils de production ou matière première. La "plus haute des solitudes 47 " se voit remiser dans les limbes de I'histoire migratoire, ou revisitée ${ }^{48}$. Les temps sont aux désirs et aux fantasmes. Aux rencontres. Amoureuses ou sexuelles. Sincères ou intéressées. Consenties ou subies. Au télescopage des cœurs et des corps. Possession. Obsessions. Avec pour toile de fond la superposition des mémoires. L'ombre de l'esclavage. De la colonisation. De l'orientalisme... L'espace est au malentendu, le temps à l'apprivoisement. Xiaolu Guo ${ }^{49}$ rapporte le journal d'une étudiante chinoise à Londres. La jeune fille y consigne les notes de sa relation amoureuse avec un Anglais pur sucre, de vingt ans son aîné. "Les choses commencent ainsi, écrit Xiaolu Guo. Par le malentendu." Quand la jeune Chinoise entend "je t'invite", elle comprend "tu m'invites de rester dans ta maison". Et elle s'installe! Une année entière où les "écarts $5^{50}$ " culturels tournent à plein : notions de respect, de travail, de carrière, d'argent, rapport au temps, à la nourriture ou à la médecine, équilibre du "je" et du "nous", et bien sûr... l'amour. Pour la jeune fille "ensemble" veut dire "ensemble", une image de la "perfection" qui se substitue à toute vie privée, la famille annihile toute considération individuelle, l'amour prend le pas sur la liberté. Aimer ne se conjugue pas, il n'appartient ni au passé ni à l'avenir, aimer est un état, une situation: "Si notre amour existait dans le temps chinois, alors, il durera toujours. Il sera infini." Et la jeune Chinoise s'emporte: "D'accord, donc l'homme et la femme paient moitié, même quand ils vivent ensemble. Et l'homme et la femme ont leur vie privée et leurs amis privés chacun. L'homme et la femme ont leurs comptes bancaires privés. Est-ce la raison pourquoi les couples occidentaux se séparent facilement et divorcent vite ${ }^{51}$ ?"

Dans Faire l'aventure, Fabienne Kanor ${ }^{22}$ raconte le périple migratoire de deux jeunes Sénégalais en Europe. Marème est une fraîche mais bêcheuse citadine qui préfère aux soupirants du cru les avances d'un journaliste français. Avec lui, elle s'envolera pour Paris où les tourtereaux convoleront. Son parcours apparaît autrement facile mais pas moins pernicieux - comparé à celui, labyrinthique, de Biram. Quand, des années après, les deux se retrouvent en Sicile, lui est resté un sanspapiers, elle a épousé un riche Italien. Le récit bat au rythme des relations sexuelles ou amoureuses - subies, volontaires, contraintes, fantasmées, instrumentalisées - entre Blancs et Noirs. Le commerce amoureux est encombré d'ambiguïtés, de représentations, d'arrière-pensées, de "perversités". Le tourisme est sexuel, le mariage de Marème utilitaire et les regards de Biram sur la Blanche Hélène empreints d'une autre "forme de perversité".

Autre rencontre, autres "rêves" et "illusions" dans Ciel de lune de Saïd Mohamed53. Récit autobio-

\footnotetext{
45. Pour reprendre le titre du roman de Rachid Mimouni, Le Fleuve détourné, Paris, Stock, 1982. 46. Ibid. 47. Tahar Ben Jelloun, La plus haute des solitudes, Paris, Seuil, 1977. 48. Voir aussi Abdellah Taïa, Un pays pour mourir, Paris, Seuil, 2015.

49. Xiaolu Guo, Petit dictionnaire chinois-anglais pour amants, traduit de l'anglais par Karine Laléchère, Paris, Buchet-Chastel 2008.

50. François Jullien, De l'universel, de l'uniforme, du commun et du dialogue entre les cultures, Paris, Fayard, 2008.

51. Xiaolu Guo, op. cit. 52. Fabienne Kanor, Faire l'aventure, Paris, JC Lattès, 2014.
} 
graphique d'un gosse placé à l'intersection du quart monde, par la branche maternelle et normande, et de l'immigration, par le père, Berbère marocain. Comme la Marème de Fabienne Kanor, la Dalila de Saïd Mohamed, fougueuse et séduisante Marocaine, aspire à changer d'air. Pour le narrateur, poète désargenté, "sang mêlé", la quête de soi se coltine encore quelques illusions identitaires doublées d'un brin d'exotisme pour la belle "Orientale". Sans le sous, le Berbéro-Normand ne sera pas la Madame de Rénal de Dalila, ce Julien Sorel en caftan. Les médisances de la vox populi et les soupçons de l'administration auront beau sermonner l'enamouré - "Votre dessein, pour vous, me fait trembler de peur; / Et de quelque façon que vous tourniez l'affaire, / Prendre femme est à vous un coup bien téméraire ${ }^{54 "}$-, rien n'y fera. Les noces seront célébrées!

Pourtant, nonobstant les malentendus et les confusions, le couple est bien réel. Et si l'un et l'autre ne regardent pas dans la même direction, n'en déplaise à la police des cœurs, le couple n'est pas une fiction. Certes, l'hymen ne sera pas une sinécure. "La seule excuse que l'on ait, c'est que le ministre de l'Intérieur ne nous a pas laissé le choix. C'est déjà plus drôle non ?" dit le narrateur goguenard face à une administration qui prétend savoir "ce qui est bon pour le citoyen". "Les lois, qui venaient d'être votées, ne libéralisaient pas la libre alliance des ressortissants de nationalités différentes. Si elle voulait rester, il fallait se marier, et il n'y avait pas à discuter." Et pour le coup, notre citoyen pourrait retourner son Molière au tartufe législateur autoproclamé directeur des consciences et des alcôves: "Oui. C'est un grand plaisir que toutes ces tendresses, / Ces propos si gentils, et ces douces caresses; / Mais il faut le goûter en toute honnêteté, I Et qu'en se mariant le crime en soit ôtés5." Aux "perversités" des mémoires et de l'Histoire de Fabienne Kanor, Saïd Mohamed ajoute la perversité du poli- tique et du législateur : ce ne sont pas les candidates à l'émigration qu'il faut blâmer, mais les tripatouillages de la loi, les immixtions du droit sur les corps et les désirs qui contraignent aux secrets, aux dissimulations, aux arrangements, voire aux mensonges et aux instrumentalisations. II ne reste trop souvent pour les unes et pour les uns qu'à "niquer la matrice56", celle des gendarmes-cerbères, police des cœurs et renifleurs de chambres à coucher. D'ailleurs, demande Alexis Jenni "que nous reste-t-il, si nous ne pouvons nous désirer, au moins du regard ? La violences7 ?"

Certes, comme Marème cherche à quitter son Sénégal natal, Dalila veut quitter le Maroc. Fuir les mœurs machistes de ses concitoyens. Pourtant, jusqu'à la dernière page, l'indécision reste entière sur cette relation : alchimie complexe de la rencontre, pièges du malentendu, mystère du lien, tout y est. Ciel de lune est un roman d'amour et d'illusions transfrontières où la parole étouffée de Dalila sur qui pèse le soupçon, referme un récit narré par le mari. Le journal de la jeune femme clôt le bec des marchands de certitudes et d'inquisition. Comme Marème. Car la jeune Sénégalaise est aussi malheureuse, insatisfaite, partagée entre un "désir obscur de moralité" et une soif universelle de bonheur. Comme est malheureuse Zahira, la prostitué marocaine dont les "yeux sont devenus tristes", mais I' "âme aspire encore au miracle58".

L'Histoire, le siècle nouveau et ses vieilles morsures, les frontières - physiques, mentales, celles en forme de voile, ou de loi - entravent la marche vers la liberté des corps et des cœurs, le droit au bonheur de "celles qui luttent, qui résistent, qui souffrent mais qui aspirent et croient toujours à la vie en assumant pleinement leur destin59", écrit Yamina Mokaddem en préface aux Carnets intimes de Taos Amrouche. "Tout revient à savoir vivre, vouloir vivre", résume la chanteuse et romancière kabyle, cette autre émigrée-immigrée, aînée dans la carrière. 